\title{
Structure of a SMG8-SMG9 complex identifies a G-domain heterodimer in the NMD effector proteins
}

\author{
LIANG LI, MAHESH LINGARAJU, CLAIRE BASQUIN, JÉROME BASQUIN, and ELENA CONTI \\ Department of Structural Cell Biology, Max-Planck-Institute of Biochemistry, D-82152 Martinsried, Germany
}

\begin{abstract}
Nonsense-mediated mRNA decay (NMD) is a eukaryotic mRNA degradation pathway involved in surveillance and posttranscriptional regulation, and executed by the concerted action of several trans-acting factors. The SMG1 kinase is an essential NMD factor in metazoans and is associated with two recently identified and yet poorly characterized proteins, SMG8 and SMG9. We determined the $2.5 \AA$ resolution crystal structure of a SMG8-SMG9 core complex from C. elegans. We found that SMG8-SMG9 is a G-domain heterodimer with architectural similarities to the dynamin-like family of GTPases such as Atlastin and GBP1. The SMG8-SMG9 heterodimer forms in the absence of nucleotides, with interactions conserved from worms to humans. Nucleotide binding occurs at the G domain of SMG9 but not of SMG8. Fitting the GDP-bound SMG8SMG9 structure in EM densities of the human SMG1-SMG8-SMG9 complex raises the possibility that the nucleotide site of SMG9 faces SMG1 and could impact the kinase conformation and/or regulation.
\end{abstract}

Keywords: NMD; post-transcriptional regulation; C. elegans; G domain

\section{INTRODUCTION}

Nonsense-mediated mRNA decay (NMD) is a eukaryotic surveillance mechanism that degrades aberrant mRNAs containing premature translation termination codons (PTCs) (Popp and Maquat 2013; Lykke-Andersen and Bennett 2014; Karousis et al. 2016). In addition, NMD is a post-transcriptional regulatory mechanism that modulates the expression of physiological mRNAs, affecting the stability of $\sim 10 \%$ of the transcriptome (Lykke-Andersen and Jensen 2015). A universal requirement for NMD is a $5^{\prime}-3^{\prime}$ RNA unwinding activity that is exerted by the helicase UPF1 and regulated by two associated factors, UPF2 and UPF3. In metazoans, UPF1 is additionally regulated by phosphorylation at the $\mathrm{N}$ - and C-terminal regions, a decisive event that creates the binding platform for recruiting SMG6 and SMG5-SMG7, which then target the transcript for degradation (Popp and Maquat 2013; Karousis et al. 2016).

UPF1 phosphorylation is catalyzed by the SMG1 kinase (Yamashita et al. 2001). In human cells, SMG1 copurifies in a complex with SMG8 and SMG9 (Yamashita et al. 2009). Human and nematode SMG8 and SMG9 proteins affect the stability of PTC-containing mRNAs in NMD reporter assays (Yamashita et al. 2009). Consistently, inhibition of human SMG-8 has been shown to ameliorate NMD-exacerbated mutant phenotypes (Usuki et al. 2013). However,

Corresponding author: conti@biochem.mpg.de

Article is online at http://www.rnajournal.org/cgi/doi/10.1261/rna.061200. 117. Freely available online through the RNA Open Access option. general impairment of NMD on natural PTC-containing targets was not detected in smg-8 mutants in C. elegans (Rosains and Mango 2012) and in human subjects carrying homozygous loss-of-function SMG9 mutations (Shaheen et al. 2016). Human patients with SMG9 deficiency display widespread transcriptional dysregulation, suggesting a predominant role of SMG9 in post-transcriptional regulation rather than in surveillance (Shaheen et al. 2016).

SMG8 and SMG9 interact with each other and inhibit the kinase activity of SMG1 in vitro (Yamashita et al. 2009; Fernández et al. 2010). Electron microscopy studies have revealed the overall architecture of the SMG1-SMG8-SMG9 complex and the central position of SMG8-SMG9 in this trimeric assembly (Arias-Palomo et al. 2011; Melero et al. 2014; Deniaud et al. 2015). However, the limited resolution of the EM maps and the absence of atomic models have so far hampered a molecular understanding of the mechanisms. In this work, we set out to obtain an atomic model of SMG8-SMG9.

\section{RESULTS AND DISCUSSION}

Using bioinformatics analyses and proteolysis experiments, we identified regions C. elegans (C.e.) full-length SMG8 (873 residues) and SMG9 (385 residues) as sufficient to form a stable heterodimeric core complex (SMG8c, residues 1-423 and SMG9c, residues 59-375, Fig. 1A) and to yield

(C) $2017 \mathrm{Li}$ et al. This article, published in $R N A$, is available under a Creative Commons License (Attribution 4.0 International), as described at http:// creativecommons.org/licenses/by/4.0/. 
diffracting crystals. After overcoming crystal lattice defects (detailed in Materials and Methods), we solved the structure and refined it at $2.5 \AA$ resolution with $R_{\text {free }}$ of $26.0 \%$ (Table 1 ).

SMG8c and SMG9c contain a similar globular fold with characteristic architecture of $\mathrm{G}$ domains along with additional secondary structure elements (Fig. 1B,C). G domains are centered at a mixed $\beta$-sheet surrounded by $\alpha$-helices on the concave and convex surfaces ( $\alpha 1, \alpha 5$ and $\alpha 2, \alpha 3, \alpha 4$, respectively) (Wittinghofer and Vetter 2011). The major structural difference between SMG8c and SMG9c is the presence in the former of a helical bundle of three C-terminal helices ( $\alpha 7-\alpha 9)$ that forms a stalk-like protrusion reminiscent of the stalk domain found in GTPases of the dynamin family, such as Atlastin and GBP1 (Fig. 1B; Supplemental Fig. S1; Daumke and Praefcke 2016).

The G domains of SMG8c and SMG9c face each other and interact with part of their convex surfaces (Fig. 1B). In particular, SMG8c helix a2A interacts with SMG9c helices a4 and $\alpha 3$ (patch 1) (in particular Val83 ${ }^{\text {SMG8 }}$, Ile86 ${ }^{\text {SMG8 }}$ with Leu258 ${ }^{\text {SMG9 }}$, Leu261 ${ }^{\text {SMG9 }}$ ) (Fig. 1D). In addition, the stalk domain of SMG8c folds back on the convex surface of SMG9c (patch 2). Here, SMG8c stalk helices $\alpha 2 B$ and $\alpha 7$ interact with SMG9c helices $\alpha 7$ and $\alpha 3$ (e.g., Ile $335^{\text {SMG8 }}$ and Phe $338^{\text {SMG8 }}$ with Val212 ${ }^{\text {SMG9 }}$ and Tyr358 ${ }^{\text {SMG9 }}$ ) (Fig. 1E). Many of the hydrophobic interface residues observed in the C. elegans SMG8c-SMG9c structure are conserved in the human orthologs (Supplemental Figs. 2, 3), suggesting a similar overall structure. To test this prediction, we engineered mutations in human full-length SMG9 (hSMG9) by substituting Met390 (corresponding to C. elegans Leu258 ${ }^{\text {SMG9 }}$ ) and Tyr515 (corresponding to C. elegans $\mathrm{Tyr} 358^{\mathrm{SMG}}{ }^{\text {) }}$. We transiently coexpressed full-length HA-tagged hSMG8 and Flag-HA-tagged hSMG9 (wild-type, M390R and M390R, Y515R mutants) in HEK293T cells and carried out coimmunoprecipitation assays with Anti-Flag affinity beads, probing with an anti-HA antibody. We found that the interaction of hSMG8 and hSMG9 observed with the wild-type proteins was indeed strongly impaired by the hSMG9 M390R mutant and almost abolished with the hSMG9 M390R, Y515R double mutant (Fig. 1F).

The relative position of the G-like domains in the SMG8cSMG9c heterodimer is remarkably similar to that observed in active dimeric GTPases of the dynamin family (Supplemental Fig. S1; Daumke and Praefcke 2016), with the two G domains converging at the loops that are known to harbor the nucleotide-binding motifs ( $G$ motifs) in canonical GTPases. However, SMG8 lacks the characteristic residues of G motifs. Another difference is that the single-stalk domain in SMG8cSMG9c has a different position as compared to the conformations observed in dynamin-like proteins (Supplemental Fig. S1; Byrnes et al. 2013). Finally, the SMG8c-SMG9c heterodimer is formed irrespective of nucleotides, while proteins such as Atlastin or GBP1 dimerize in the presence of GTP analogs (Ghosh et al. 2006; Bian et al. 2011; Byrnes and Sondermann 2011).
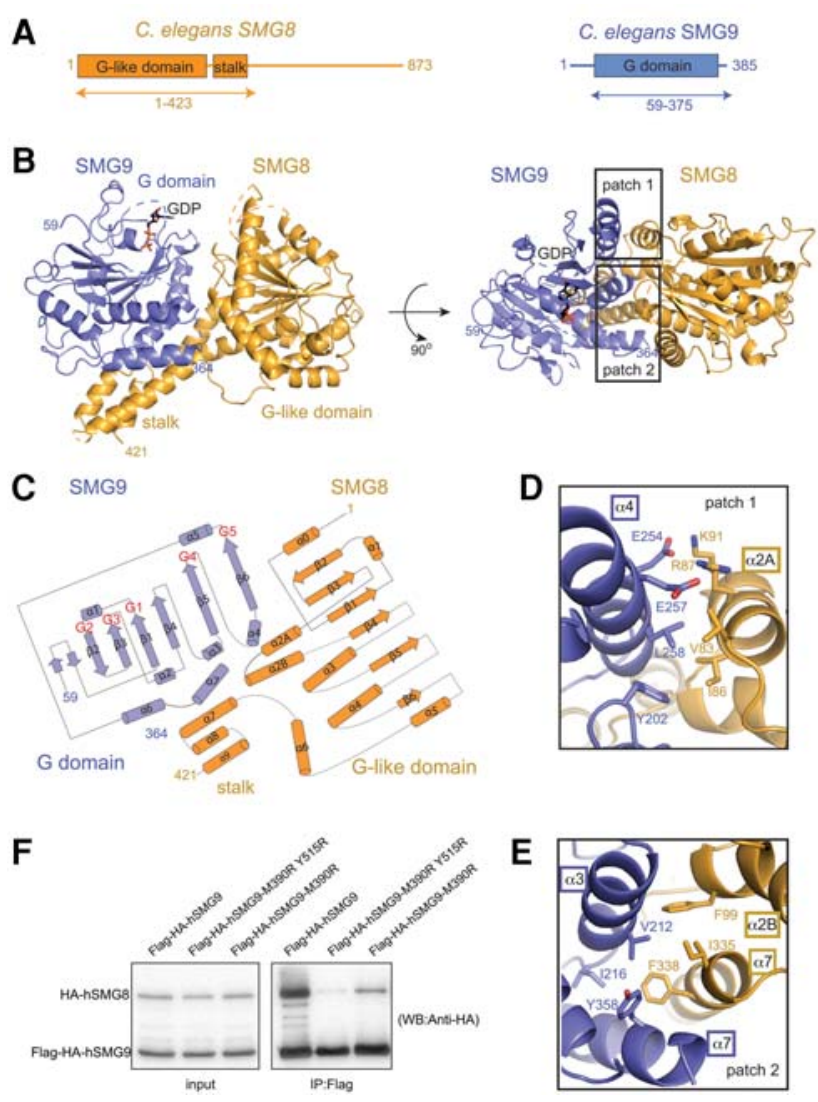

FIGURE 1. Structure of the conserved core of C. elegans SMG8-SMG9. (A) Schematic representation of the domain organization of $C$. elegans SMG8 (in orange) and SMG9 (in blue). Domains with a structured fold are shown as rectangles and labeled. Predicted low-complexity regions are shown as lines. The arrows below the diagram highlight the parts of the proteins that were crystallized. $(B)$ Two views of the crystal structure of the C. elegans SMG8c-SMG9c core complex, with the molecules shown in orange and blue, respectively. The two views are related by a $90^{\circ}$ clockwise rotation around a horizontal axis. The G-like domains and the stalk domain are indicated, as well as the $\mathrm{N}$ - and C-terminal residues with ordered electron density. The GDP moiety bound to the SMG9 G domain is shown in stick representation. Disordered loops are highlighted with dotted lines. On the right, the two rectangles highlight the two main interaction interfaces (patches 1 and 2) that are shown in more detail below in panels $D$ and $E$. (C) Topological diagram of SMG8c and SMG9c ( $\beta$-strands shown as arrows and $\alpha$-helices as cylinders). Loops between secondary SMG9 feature similarities in the socalled $\mathrm{G}$ motifs as compared to other $\mathrm{G}$ domains. The positions of the $\mathrm{G}$ motifs in the loops between secondary structure elements are indicated in red. Note that SMG8c and SMG9c feature additional elements as compared to canonical $\mathrm{G}$ domains ( $\alpha 2 \mathrm{~A}, \alpha 6$ and $\alpha 6, \alpha 7$, respectively). $(D)$ Zoomed-in view of the interacting residues at patch 1 . The molecule is shown after $\sim 180^{\circ}$ rotation around a horizontal axis with respect to the view in panel $A$. SMG8c helix $\alpha 2 A$ and SMG9c helices $\alpha 3$ and $\alpha 4$ are labeled. $(E)$ Zoomed-in view of the interacting residues at patch 2 . The molecule is shown in a similar orientation as in panel $C$. SMG8c stalk helices $\alpha 2 \mathrm{~B}$ and $\alpha 7$ and SMG9c helices $\alpha 3$ and $\alpha 7$ are indicated. $(F)$ Coimmunoprecipitation assays of human full-length HA-tagged hSMG8 and Flag-HA-tagged hSMG9 (wild-type or mutants) in transiently transfected HEK293T cells. Cell lysates (input) were immunoprecipitated with Flag binder and detected with an HA-antibody (precipitate) (12\% SDS-PAGE gel). The mutated residues in human SMG9 (M390 and Y515) correspond to C. elegans SMG9 Leu258 (patch 1, panel $D$ ) and Tyr358 (patch 2, panel E). HA-SMG8 is $111.7 \mathrm{kDa}$ and Flag-HA-SMG9 is $63.6 \mathrm{kDa}$. 
TABLE 1. Data collection and refinement statistics

\begin{tabular}{|c|c|c|}
\hline & CeSMG8-9-Apo & CeSMG8-9-GDP \\
\hline \multicolumn{3}{|l|}{ Data set } \\
\hline Wavelength $(\AA)$ & 0.979 & 0.9785 \\
\hline Resolution range $(\AA)^{\mathrm{a}}$ & $52.36-2.493(2.583-2.493)$ & $47.48-2.65(2.734-2.65)$ \\
\hline Space group & P 3221 & P 3221 \\
\hline$a, b, c(\AA)$ & $111.085,111.085,374.474$ & $110.605,110.605,360.266$ \\
\hline$\alpha, \beta, \gamma\left(^{\circ}\right)$ & 9090120 & 9090120 \\
\hline Total reflections $\mathrm{s}^{\mathrm{a}}$ & $3,768,766(360,260)$ & $764,628(71,339)$ \\
\hline Unique reflections $^{\mathrm{a}}$ & $94,467(9210)$ & $76,172(7433)$ \\
\hline Multiplicity $^{\mathrm{a}}$ & $39.9(39.1)$ & $10.0(9.6)$ \\
\hline Completeness $(\%)^{\mathrm{a}}$ & $100(98)$ & $100(99)$ \\
\hline Mean $I / \operatorname{sigma}(I)^{\mathrm{a}}$ & $27.25(1.61)$ & $12.39(1.85)$ \\
\hline $\mathrm{R}-$ merge $^{\mathrm{a}}$ & $0.1374(2.587)$ & $0.1198(1.09)$ \\
\hline $\mathrm{CC} 1 / 2^{\mathrm{a}}$ & $1(0.783)$ & $0.998(0.793)$ \\
\hline \multicolumn{3}{|l|}{ Refinement } \\
\hline R-work & 0.2247 & 0.2318 \\
\hline R-free & 0.2602 & 0.2740 \\
\hline Average B-factor & 89.31 & 81.5 \\
\hline Ligands & & GDP, Mg, EDO \\
\hline \multicolumn{3}{|l|}{ Stereochemistry } \\
\hline RMS (bonds) & 0.003 & 0.003 \\
\hline RMS (angles) & 0.57 & 0.72 \\
\hline Ramachandran favored (\%) & 96 & 96 \\
\hline Ramachandran allowed (\%) & 3.6 & 4.1 \\
\hline Ramachandran outliers (\%) & 0 & 0 \\
\hline
\end{tabular}

${ }^{a}$ Values in parentheses correspond to the highest-resolution shell. binding. There are two notable differences in the G1-G3 motifs of SMG9c as compared to the dynamin-like family. First, there is a conserved proline residue (Pro153 ${ }^{\mathrm{SMG9}}$, disordered in the present structure) at the position of switch 2 typically occupied by a glycine (Fig. 2B,C). Second, there is a conserved glycine residue $\left(\right.$ Gly96 ${ }^{\text {SMG9 }}$ ) in the $\mathrm{P}$ loop at the equivalent position of the so-called arginine "finger" (Arg77 ${ }^{\text {Atlastin }}$ ) (Fig. 2B,C). Consistent with the absence of such arginine (which stimulates the GTPase activity of dynamin-like proteins in cis), we did not detect convincing GTPase hydrolysis in vitro (data not shown). Another significant difference is at the G4 and G5 loops that bind the base of the nucleotide in dynamin-like proteins. The characteristic guanosine specificity determinant of Atlastin and GBP1 in the G4 motif is not present in SMG9 (Fig. 2B,C). At the corresponding position of Asp218 ${ }^{\text {Atlastin }}$, SMG9 features a conserved lysine residue (Lys241 ${ }^{\text {SMG9 }}$ ) that stacks with its aliphatic portion on top of the guanine base. With the caveat that motif G5 is largely disordered, none of the interactions in the current

We tested whether SMG8c-SMG9c can bind guanosine nucleotides. In fluorescence binding assays with mantnucleotide derivatives, mant-GDP bound SMG8c-SMG9c and SMG9c with a dissociation constant $\left(K_{\mathrm{d}}\right)$ of $10 \mu \mathrm{M}$ and $15 \mu \mathrm{M}$, respectively (Fig. 2A). Mant-GTP $\gamma \mathrm{S}$ bound SMG8c-SMG9c with a $K_{\mathrm{d}}$ of $6.5 \mu \mathrm{M}$, suggesting a slightly tighter binding in the presence of the nucleotide $\gamma$-phosphate (Fig. 2A). In general, the low-micromolar binding affinities we measured for SMG8c-SMG9c are similar to those reported for GBP1 (Praefcke et al. 1999). We proceeded to obtain the structure of a nucleotide-bound SMG8c-SMG9c complex. Although the SMG8c-SMG9c crystals cracked when soaking GTP, GDP soaking experiments were successful. Diffraction data to $2.65 \AA$ resolution (Table 1) showed the presence of well-defined electron density for a GDP moiety in SMG9c but not in SMG8c (Supplemental Fig. S4).

GDP binds SMG9c at a similar position as in Atlastin and GBP1, in particular with similarities at the phosphatebinding loops, e.g., at the motifs G1 (P loop), G2 (switch 1), and G3 (switch 2) (Fig. 2B,C). In SMG9c, the P loop residues Lys99 ${ }^{\text {SMG9 }}$ and Ser $100^{\text {SMG9 }}$ coordinate the phosphates of GDP. Although parts of the switch regions are disordered in our GDP-bound structure, the switch 2 residue Asp $150^{\text {SMG9 }}$ is at the position expected for coordinating the magnesium ion, while the switch 1 residue $\mathrm{Thr} 135^{\text {SMG9 }}$ is $10 \AA$ away from the position expected upon $\gamma$-phosphate structure engage guanine-specific moieties.

We used our coordinates to progress in the interpretation of cryo-EM structures of human SMG1-SMG8-SMG9 that have been recently resolved at $\sim 20 \AA$ resolution (Fig. 3; Arias-Palomo et al. 2011; Melero et al. 2014; Deniaud et al. 2015). We fitted a homology model of SMG1 with the kinase domain in the "head" region of the density and the N-terminal HEAT-repeat domain in the "arm" region, as in Deniaud et al. (2015). We positioned the C. elegans SMG8c-SMG9c structure in the remaining unoccupied density that is connected to the "arm," in a density previously shown to correspond to human SMG8-SMG9 (Arias-Palomo et al. 2011). Although the interpretation of low-resolution maps needs to be judged with caution, placing the atomic coordinates appeared to result in a remarkably good fit, whereby the G domain of SMG9 is at the center of the density, with the G-motif loops pointing toward the HEAT repeat region of SMG1 (Fig. 3). In this pseudo-atomic model, SMG8 has a more peripheral position, with the G-like domain approaching the N-terminal end of the "arm" while the stalk is exposed to solvent.

This pseudo-atomic model is generally in agreement with previous biochemical data (Yamashita et al. 2009; Deniaud et al. 2015). The start of the G domain of C. elegans SMG9 (residue 59) is near the density of the SMG1 HEAT repeat "arm." Consistently, the low-complexity $\mathrm{N}$-terminal region 
of human SMG9 has been shown to interact with the SMG1 HEAT-repeat domain in co-IP assays (Yamashita et al. 2009) and in crosslinking-mass spectrometry experiments (Deniaud et al. 2015). The end of the folded domain of C. elegans SMG8 (residue 421) points toward the SMG1 C-termi-

A

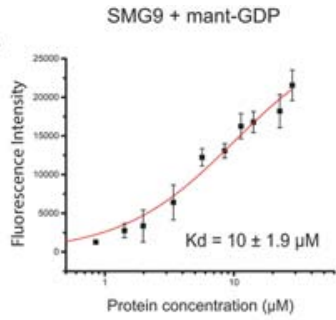

B

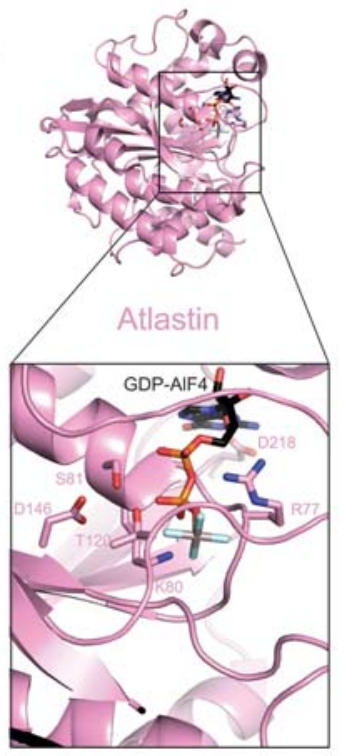

C

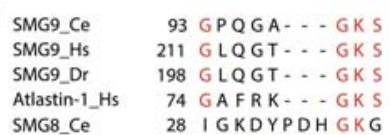

G 1
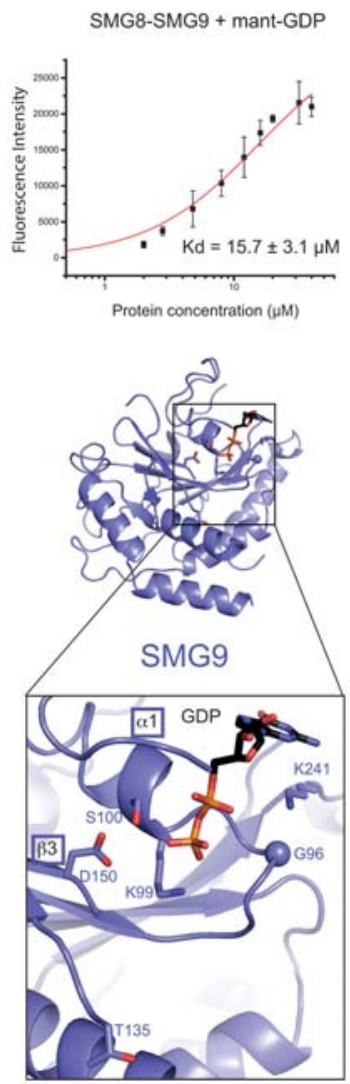

\begin{abstract}
128 NEQSRHQT I 246 KERGGNQTS 233 KERAGNQSS 113 RGGSERETT 52 VAATEDETC
\end{abstract} G 2
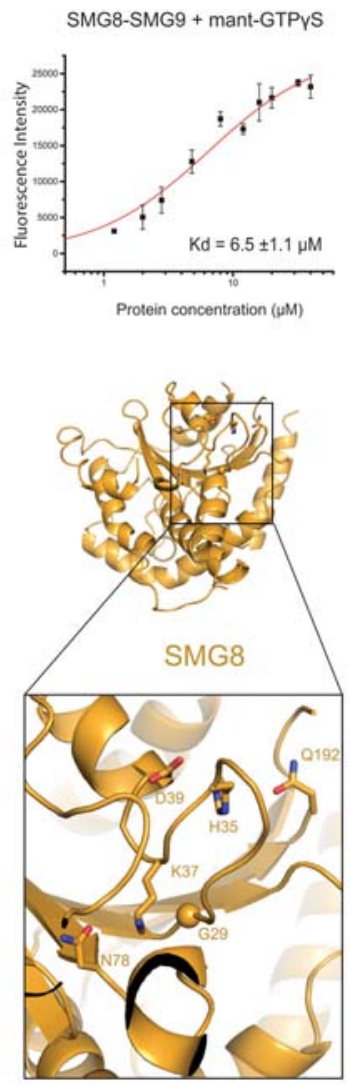

150 DCQP 269 DTQP 256 DTQP 146 DTQ

G 3 nal "head." Consistently, the low-complexity C-terminal region of human SMG8 has been shown to contact an insertion domain present in the C-terminal domain of human SMG1 (Deniaud et al. 2015). Finally, the $\beta 5-\alpha 4$ loop of SMG8 faces the density of the SMG1 N-terminal arch. Consistently, the corresponding loop of human SMG8 (residues 290-293) has been shown to contact the SMG1 $\mathrm{N}$ terminus in crosslinking-mass spectrometry experiments (Deniaud et al. 2015). Although parts of the SMG9 G motifs as well as the low-complexity regions described above are not present in the current SMG8c-SMG9c crystal structure, the fitting suggests that they might become ordered upon SMG1 binding. In summary, the pseudo-atomic model not only rationalizes how SMG9 recruits the more peripheral SMG8 to the SMG1 complex (Deniaud et al. 2015), but also has predictive value because it raises the hypothesis that the nucleotide-binding state of SMG9 might impact on the entire complex.

\section{MATERIALS AND METHODS}

\section{Protein expression and purification}

We analyzed the amino acid sequence of SGM8 and SMG9 proteins from different species in an effort to identify orthologs that would be best suited for crystallization. We selected the C. elegans (C.e.) proteins since they are $10 \%-25 \%$ smaller and therefore likely more compact than their human counterparts. C.e. SMG8 (873 residues) and SMG9 (385 residues) were subcloned from a C.e. cDNA library with standard PCR protocols in a single MultiBac expression vector ( $\mathrm{pFL}$ ) (Fitzgerald et al. 2006). SMG8 was cloned into the multiple cloning site 1 (MCS1) of the pFL vector using Xmal and Nhe1, while SMG9 was cloned into the multiple cloning site 2 (MCS2) using BamHI and SalI. Coexpression was crucial to obtain the heterodimer: Although SMG9 could be expressed and purified in a soluble form, SMG8 was insoluble when in isolation (data not shown). Rounds of limited proteolysis and optimization of the expression constructs narrowed down the SMG8c-SMG9c core complex (C.e. SMG8 1-423 and SMG9 59-375). SMG8c-SMG9c were coexpressed in baculovirus-infected Hi-Five insect cells (Invitrogen) at $26^{\circ} \mathrm{C}$ for $70 \mathrm{~h}$. Cells were lysed in $25 \mathrm{mM}$ Tris pH 8.0 with $300 \mathrm{mM} \mathrm{NaCl}$, 


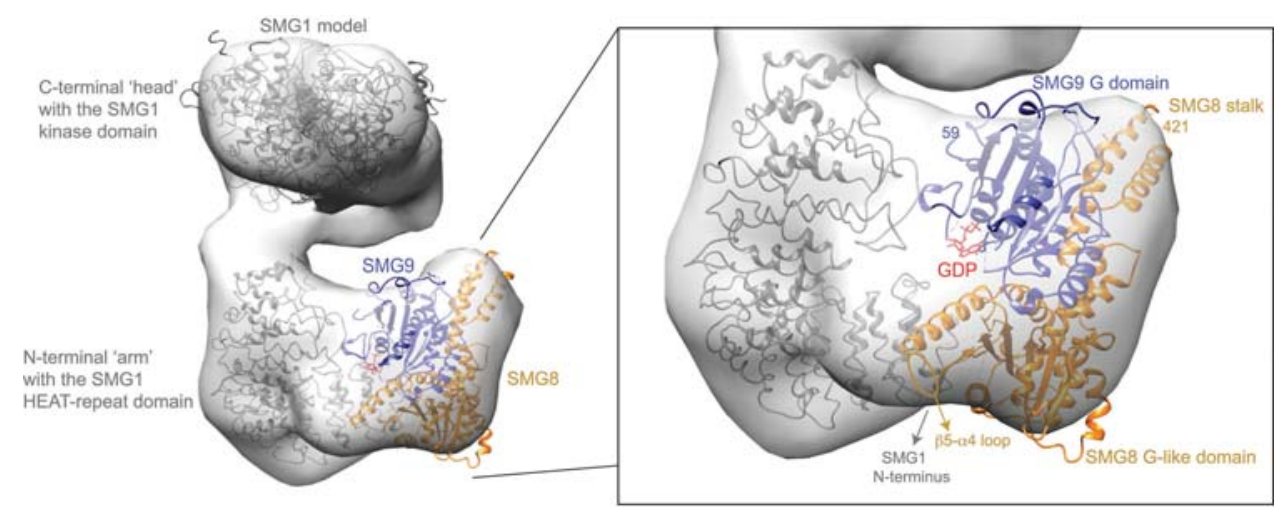

FIGURE 3. Pseudo-atomic model of a SMG1-SMG8-SMG9 complex. In gray is the EM density of a human SMG1-SMG8-SMG9 complex fitted with a model of human SMG1, as in Deniaud et al. (2015). The remaining density was fitted with the crystal structure of the C. elegans SMG8SMG9 core complex (which lacks the low-complexity SMG9 N-terminal and SMG8 C-terminal regions). In red is the GDP molecule bound to SMG9. The fitting was done in Chimera (Pettersen et al. 2004).

and $20 \mathrm{mM}$ imidazole was supplemented to the supernatant before loading onto the nickel column. The complex was purified by nickel-based affinity chromatography via a C-terminal hexahistidine tag on C.e. SMG8, and subsequent ion exchange (Heparin HiTrap) and gel-filtration chromatography (Superdex200, equilibrated with $25 \mathrm{mM}$ Tris, $300 \mathrm{mM} \mathrm{NaCl}, \mathrm{pH}$ 8.0). SelenoMethionine (SeMet) substituted proteins were expressed in insect cells with similar protocols that we reported previously (Halbach et al. 2013). The purification procedure of the SeMet-substituted complex was the same as for the native protein, except that all buffers were degassed and $4 \mathrm{mM} \beta$-mercaptoethanol and $2 \mathrm{mM}$ DTT were added before and after elution from the $\mathrm{Ni}^{2+}-\mathrm{NTA}$ resin, respectively. Mass spectrometry analysis showed the presence of $\sim 60 \%$ SeMet incorporation in the purified complex.

\section{Crystallization and structure determination}

C.e. SMG8c-CeSMG9c crystallized by vapor diffusion in several PEG conditions at $\mathrm{pH} 8.0$ and $10^{\circ} \mathrm{C}$. These initial crystals diffracted to $\sim 3.0 \AA$ resolution and could be processed in a hexagonal spacegroup, but analysis of the cumulative intensity distribution showed the presence of merohedral twinning with a twin fraction close to 0.5 . Additive screening allowed us to identify yttrium chloride as an effective chemical compound to overcome the twinning problem. The best un-twinned crystals were grown by hanging-drop vapor diffusion in drops formed by equal volumes $(1.5 \mu \mathrm{L})$ of protein $(6.8 \mathrm{mg} / \mathrm{mL}$ in gel filtration buffer supplemented with $0.11 \mathrm{mM}$ $\mathrm{YCl}_{3}$ ) and crystallization buffer (10\% PEG3350, $0.1 \mathrm{M}$ Tris $\mathrm{pH}$ 8.5). SeMet crystals were obtained using the same conditions, but adding tris(2-carboxyethyl)phosphine (TCEP, to limit SeMet oxidation) and covering the reservoir buffer with paraffin oil (to slow drop evaporation and increase crystal size). All crystals were cryoprotected with the crystallization buffer supplemented with $25 \%$ ethylene glycol prior to cryo-cooling and data collection.

Diffraction data were collected at $100 \mathrm{~K}$ at the Swiss Light Source (SLS) beamline PXII. Diffraction data were collected at the selenium K-edge peak wavelength and were processed with XDS (Kabsch 2010). The crystals belong to a trigonal P3221 space group with three copies of the complex in the asymmetric unit related by noncrystallographic symmetry. We used SHELX for phasing (Sheldrick
2010) and phenix.autobuild for initial model building (Adams et al. 2010). We completed the model with iterative rounds of manual building in Coot and refinement with phenix.refine. The three independent copies of the complex in the asymmetric unit are very similar and contain most of the polypeptide chains, except disordered loop regions. The copy of SMG8c-SMG9c described in the text contains SMG8 residues 1-416 (with the exception of disordered loops between residues 193-211, 256-288, and 356-386) and SMG9 residues 59-363 (with the exception of disordered loops between residues 124-134, 152-172, and 284-311) (Table 1).

Native crystals were soaked with $10 \mathrm{mM}$ GDP for $5 \mathrm{~min}$ prior to freezing. The structure of C.e. SMG8c-SMG9c-GDP was determined by molecular replacement with Phaser using the SeMet-derivatized CeSMG8-9 structure as a search model. The model was completed with Coot (Emsley et al. 2010) and refined with phenix.refine (Adams et al. 2010).

\section{Nucleotide-binding experiments}

The affinities for GDP were determined by fluorescence measurements on an Infinite M1000 Pro (Tecan). Experiments were carried out at $21^{\circ} \mathrm{C}$ in a buffer containing $25 \mathrm{mM}$ Tris pH 8.5, $150 \mathrm{mM} \mathrm{NaCl}$, and $5 \mathrm{mM} \mathrm{MgCl}_{2}$. Increasing protein concentrations were incubated with $1.67 \mu \mathrm{M}$ of methylanthraniloyl (mant) labeled GDP for $30 \mathrm{~min}$ at room temperature. The experiments were carried out with the fragments crystallized, since the full-length proteins were prone to degradation of the low complexity sequences. Fluorescence of mant-GDP was excited at $355 \mathrm{~nm}$ and emission spectra were then monitored from 400 to $500 \mathrm{~nm}$, with emission maxima detected at $448 \mathrm{~nm}$. The intrinsic protein fluorescence as well as the mant-nucleotide background was subtracted from the curves. Curve fittings were done with Origin with a one-to-one binding model and are consistent with the presence of one molecule of nucleotide per heterodimer. Curves were done in triplicate. Similar approaches were used to determine the binding affinities for GTP $\gamma \mathrm{S}$.

\section{Coimmunoprecipitation assays}

Both the SMG8 and SMG9 were cloned in a vector containing the EF-1 a promoter and with an N-terminal Flag tag and N-terminal 
HA tag using EcoRI and NotI restriction sites. HEK293T cells were cultured in Dulbecco's modified Eagle medium containing 10\% fetal bovine serum (Gibco), $100 \mathrm{U} / \mathrm{mL}$ penicillin, and $0.1 \mathrm{mg} / \mathrm{mL}$ streptomycin (Gibco) at $32^{\circ} \mathrm{C} / 5 \% \mathrm{CO}_{2}$. Plasmids were transfected with polyethyleneimine (Polysciences Inc., $1 \mathrm{mg} / \mathrm{mL}$ ) for protein interaction studies. HEK293T cells were collected from confluent six-well plates after $72 \mathrm{~h}$ of transient transfection. Cells were lysed in $0.5 \mathrm{~mL}$ of lysis buffer containing $50 \mathrm{mM}$ Tris, $\mathrm{pH} 7.4,150 \mathrm{mM}$ $\mathrm{NaCl}$, supplemented with protease inhibitor cocktail (Roche) and DNase I. The lysate was centrifuged at $16,000 \mathrm{~g}$ for $30 \mathrm{~min}$ at $4^{\circ} \mathrm{C}$. Twelve microliters of Anti-Flag M2 sepharose beads (Sigma) were added to supernatant for $1 \mathrm{~h}$ at $4^{\circ} \mathrm{C}$. Beads were washed four times with $1 \mathrm{~mL}$ of buffer containing $50 \mathrm{mM}$ Tris, pH 7.4, $300 \mathrm{mM} \mathrm{NaCl}$, and proteins were eluted with $25 \mu \mathrm{L}$ of lysis buffer supplemented with $100 \mu \mathrm{g} / \mathrm{mL}$ flag peptide (Sigma-Aldrich, F3290). Eluted proteins were run on $12 \%$ polyacrylamide gels and transferred onto polyvinylidene difluoride membrane $(0.45 \mu \mathrm{m}$ pore size $)$ (Millipore Immobilon-P) for Western blotting. Anti-HA (Covance, MMS-101 R) antibody and horseradish peroxidasecoupled goat anti-mouse (Millipore, AQ502A) secondary antibody were used in combination with ECL prime Western blotting detection reagent (GE healthcare) for detection of Flag-HA and HA-tagged proteins via Western blotting.

\section{DATA DEPOSITION}

The coordinates have been deposited in the Protein Data Bank with accession codes 5NKM (SMG8-SMG9) and 5NKK (SMG8SMG9-GDP).

\section{SUPPLEMENTAL MATERIAL}

Supplemental material is available for this article.

\section{ACKNOWLEDGMENTS}

We thank the staff of the MPIB Crystallization Facility for robotic crystal screening and optimization setups and of the Core Facility of MPIB for mass spectrometry analysis. We thank Christiane Schaffitzel for the coordinates of the human SMG1 model (Deniaud et al. 2015). We also thank the staff of the PX beamlines of the SLS synchrotron for assistance in data collection. This study was supported by the Max Planck Gesellschaft, the European Commission (ERC Advanced Investigator Grant 294371 and Marie Curie ITN RNPnet), and the Deutsche Forschungsgemeinschaft (DFG SFB646, SFB1035, GRK1721, FOR1680, and CIPSM) to E.C.

Author contributions: L.L. purified, crystallized, and solved the structure together with J.B.; M.L. carried out the co-IP assays; C.B. carried out biophysical and biochemical assays; E.C. and J.B. supervised the project; E.C. and L.L. wrote the manuscript.

Received February 22, 2017; accepted April 4, 2017.

\section{REFERENCES}

Adams PD, Afonine PV, Bunkóczi G, Chen VB, Davis IW, Echols N, Headd JJ, Hung L-W, Kapral GJ, Grosse-Kunstleve RW, et al. 2010. PHENIX: a comprehensive Python-based system for macro- molecular structure solution. Acta Crystallogr D Biol Crystallogr 66: 213-221.

Arias-Palomo E, Yamashita A, Fernández IS, Núñez-Ramírez R, Bamba Y, Izumi N, Ohno S, Llorca O. 2011. The nonsense-mediated mRNA decay SMG-1 kinase is regulated by large-scale conformational changes controlled by SMG-8. Genes Dev 25: 153-164.

Bian X, Klemm RW, Liu TY, Zhang M, Sun S, Sui X, Liu X, Rapoport TA, Hu J. 2011. Structures of the atlastin GTPase provide insight into homotypic fusion of endoplasmic reticulum membranes. Proc Natl Acad Sci 108: 3976-3981.

Byrnes LJ, Sondermann H. 2011. Structural basis for the nucleotide-dependent dimerization of the large $\mathrm{G}$ protein atlastin-1/SPG3A. Proc Natl Acad Sci 108: 2216-2221.

Byrnes LJ, Singh A, Szeto K, Benvin NM, O’Donnell JP, Zipfel WR, Sondermann H. 2013. Structural basis for conformational switching and GTP loading of the large G protein atlastin. EMBO $J$ 32: 369-384.

Daumke O, Praefcke GJK. 2016. Invited review: mechanisms of GTP hydrolysis and conformational transitions in the dynamin superfamily. Biopolymers 105: 580-593.

Deniaud A, Karuppasamy M, Bock T, Masiulis S, Huard K, Garzoni F, Kerschgens K, Hentze MW, Kulozik AE, Beck M, et al. 2015. A network of SMG-8, SMG-9 and SMG-1 C-terminal insertion domain regulates UPF1 substrate recruitment and phosphorylation. Nucleic Acids Res 43: 7600-7611.

Emsley P, Lohkamp B, Scott WG, Cowtan K. 2010. Features and development of Coot. Acta Crystallogr D Biol Crystallogr 66: 486-501.

Fernández IS, Yamashita A, Arias-Palomo E, Bamba Y, Bartolomé RA, Canales MA, Teixidó J, Ohno S, Llorca O. 2010. Characterization of SMG-9, an essential component of the nonsense-mediated mRNA decay SMG1C complex. Nucleic Acids Res 39: 347-358.

Fitzgerald DJ, Berger P, Schaffitzel C, Yamada K, Richmond TJ, Berger I. 2006. Protein complex expression by using multigene baculoviral vectors. Nat Methods 3: 1021-1032.

Ghosh A, Praefcke GJK, Renault L, Wittinghofer A, Herrmann C. 2006. How guanylate-binding proteins achieve assembly-stimulated processive cleavage of GTP to GMP. Nature 440: 101-104.

Halbach F, Reichelt P, Rode M, Conti E. 2013. The yeast ski complex: crystal structure and RNA channeling to the exosome complex. Cell 154: 814-826.

Kabsch W. 2010. Integration, scaling, space-group assignment and postrefinement. Acta Crystallogr D Biol Crystallogr 66: 133-144.

Karousis ED, Nasif S, Mühlemann O. 2016. Nonsense-mediated mRNA decay: novel mechanistic insights and biological impact. WIREs RNA 7: 661-682.

Lykke-Andersen J, Bennett EJ. 2014. Protecting the proteome: eukaryotic cotranslational quality control pathways. J Cell Biol 204: 467-476.

Lykke-Andersen S, Jensen TH. 2015. Nonsense-mediated mRNA decay: an intricate machinery that shapes transcriptomes. Nat Rev Mol Cell Biol 16: 665-677.

Melero R, Uchiyama A, Castaño R, Kataoka N, Kurosawa H, Ohno S, Yamashita A, Llorca O. 2014. Structures of SMG1-UPFs complexes: SMG1 contributes to regulate UPF2-dependent activation of UPF1 in NMD. Structure 22: 1105-1119.

Pettersen EF, Goddard TD, Huang CC, Couch GS, Greenblatt DM, Meng EC, Ferrin TE. 2004. UCSF Chimera-a visualization system for exploratory research and analysis. J Comput Chem 25: 1605-1612.

Popp MW-L, Maquat LE. 2013. Organizing principles of mammalian nonsense-mediated mRNA decay. Annu Rev Genet 47: 139-165.

Praefcke GJ, Geyer M, Schwemmle M, Robert Kalbitzer H, Herrmann C. 1999. Nucleotide-binding characteristics of human guanylate-binding protein 1 (hGBP1) and identification of the third GTP-binding motif. J Mol Biol 292: 321-332.

Rosains J, Mango SE. 2012. Genetic characterization of smg-8 mutants reveals no role in C. elegans nonsense mediated decay. PLoS ONE 7: e49490. 


\section{Li et al.}

Shaheen R, Anazi S, Ben-Omran T, Seidahmed MZ, Caddle LB, Palmer K, Ali R, Alshidi T, Hagos S, Goodwin L, et al. 2016. Mutations in SMG9, encoding an essential component of nonsense-mediated decay machinery, cause a multiple congenital anomaly syndrome in humans and mice. Am J Hum Genet 98: 643-652.

Sheldrick GM. 2010. Experimental phasing with SHELXC/D/E: combining chain tracing with density modification. Acta Crystallogr D Biol Crystallogr 66: 479-485.

Usuki F, Yamashita A, Shiraishi T, Shiga A, Onodera O, Higuchi I, Ohno S. 2013. Inhibition of SMG-8, a subunit of SMG-1 kinase, ameliorates nonsense-mediated mRNA decay-exacerbated mutant phenotypes without cytotoxicity. Proc Natl Acad Sci 110: 15037-15042.
Wittinghofer A, Vetter IR. 2011. Structure-function relationships of the G domain, a canonical switch motif. Biochemistry 80: 943-971.

Yamashita A, Ohnishi T, Kashima I, Taya Y, Ohno S. 2001. Human SMG-1, a novel phosphatidylinositol 3-kinase-related protein kinase, associates with components of the mRNA surveillance complex and is involved in the regulation of nonsense-mediated mRNA decay. Genes Dev 15: 2215-2228.

Yamashita A, Izumi N, Kashima I, Ohnishi T, Saari B, Katsuhata Y, Muramatsu R, Morita T, Iwamatsu A, Hachiya T, et al. 2009. SMG-8 and SMG-9, two novel subunits of the SMG-1 complex, regulate remodeling of the mRNA surveillance complex during nonsense-mediated mRNA decay. Genes Dev 23: 1091-1105. 

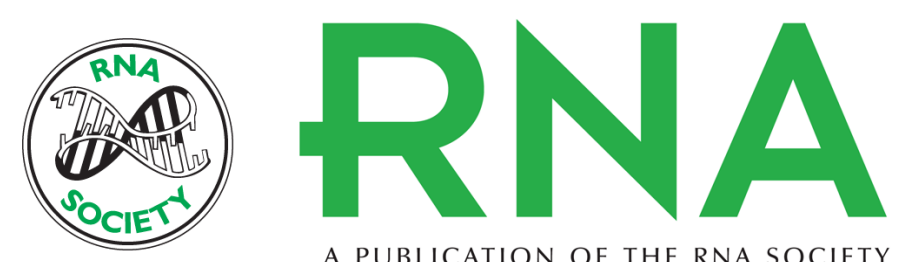

A PUBLICATION OF THE RNA SOCIETY

\section{Structure of a SMG8-SMG9 complex identifies a G-domain heterodimer in the NMD effector proteins}

Liang Li, Mahesh Lingaraju, Claire Basquin, et al.

RNA 2017 23: 1028-1034 originally published online April 7, 2017

Access the most recent version at doi:10.1261/rna.061200.117

\section{Supplemental http://rnajournal.cshlp.org/content/suppl/2017/04/07/rna.061200.117.DC1 \\ Material}

References This article cites 27 articles, 8 of which can be accessed free at: http://rnajournal.cshlp.org/content/23/7/1028.full.html\#ref-list-1

Open Access Freely available online through the RNA Open Access option.

Creative This article, published in RNA, is available under a Creative Commons License Commons (Attribution 4.0 International), as described at

License http://creativecommons.org/licenses/by/4.0/.

Email Alerting Receive free email alerts when new articles cite this article - sign up in the box at the Service top right corner of the article or click here. 